\title{
Some Remarks on 1D Schrödinger Operators With Localized Magnetic and Electric Potentials
}

\author{
Yuriy Golovaty* \\ Department of Differential Equations, Ivan Franko National University of Lviv, Lviv, Ukraine
}

One-dimensional Schrödinger operators with singular perturbed magnetic and electric potentials are considered. We study the strong resolvent convergence of two families of the operators with potentials shrinking to a point. Localized $\delta$-like magnetic fields are combined with $\delta^{\prime}$-like perturbations of the electric potentials as well as localized rank-two perturbations. The limit results obtained heavily depend on zero-energy resonances of the electric potentials. In particular, the approximation for a wide class of point interactions in one dimension is obtained.

Keywords: 1D Schrödinger operator, magnetic potential, zero-energy resonance, half-bound state, short range interaction, point interaction, $\delta$-potential, $\delta^{\prime}$-potential

\section{OPEN ACCESS}

Edited by:

Manuel Gadella

University of Valladolid, Spain

Reviewed by:

Hernando Quevedo,

National Autonomous University of

Mexico, Mexico

Alberto Alonso-Izquierdo,

University of Salamanca, Spain

*Correspondence:

Yuriy Golovaty

yuriy.golovaty@Inu.edu.ua

Specialty section: This article was submitted to Mathematical Physics, a section of the journal

Frontiers in Physics

Received: 17 November 2018 Accepted: 18 April 2019

Published: 07 May 2019

Citation:

Golovaty Y (2019) Some Remarks on 1D Schrödinger Operators With

Localized Magnetic and Electric Potentials. Front. Phys. 7:70. doi: 10.3389/fphy.2019.00070
2000 Mathematics Subject Classication: Primary 34L40, 34E15; Secondary 81Q10.

\section{INTRODUCTION}

The present paper is concerned with the convergence of families of singularly perturbed one-dimensional magnetic Schrödinger operators. Our motivation of the study on this convergence comes from an application to the scattering of quantum particles by sharply localized potentials and finite rank perturbations. The main purpose is to construct solvable models in terms of the point interactions describing with admissible fidelity the real quantum interactions. The Schrödinger operators with potentials that are distributions supported on discrete sets (such potentials are usually termed point interactions) have attracted considerable attention since the 1980s. It is an extensive subject with a large literature (see $[1,2]$, and the references given therein).

It is well-known that all nontrivial point interactions at a point $x$ can be described by the coupling conditions

$$
\left(\begin{array}{c}
\psi(x+0) \\
\psi^{\prime}(x+0)
\end{array}\right)=e^{i \varphi}\left(\begin{array}{ll}
c_{11} & c_{12} \\
c_{21} & c_{22}
\end{array}\right)\left(\begin{array}{c}
\psi(x-0) \\
\psi^{\prime}(x-0)
\end{array}\right)
$$

where $\varphi \in\left[-\frac{\pi}{2}, \frac{\pi}{2}\right], c_{k l} \in \mathbb{R}$, and $c_{11} c_{22}-c_{12} c_{21}=1$ (see, e.g., $[3,4]$ ). The nontriviality of point interactions means that the associated self-adjoint operator cannot be presented as a direct sum of two operators acting in $L_{2}(-\infty, 0)$ and $L_{2}(0, \infty)$. For the quantum systems described by the Schrödinger operators with regular potentials localized in a neighborhood of $x$ one can often assign the Schrödinger operators with the point interactions (1.1) so that the corresponding zero-range models govern the quantum dynamics of the true interactions with adequate accuracy, especially for the low-energy particles. In this context, the inverse problem is also of interest. The important question is how to approximate a given point interaction by Schrödinger operators with localized regular potentials or finite-rank perturbations. 
We study the families of the Schrödinger operators that can be partially viewed as regularizations of the pseudo-Hamiltonians

$$
\begin{gathered}
\left(i \frac{d}{d x}+a \delta(x)\right)^{2}+b \delta^{\prime}(x)+c \delta(x) \\
\left(i \frac{d}{d x}+a \delta(x)\right)^{2}+b\left(\left\langle\delta^{\prime}(x), \cdot\right\rangle \delta(x)+\langle\delta(x), \cdot\rangle \delta^{\prime}(x)\right)+c \delta(x)
\end{gathered}
$$

where $\delta$ is Dirac's delta function. We note that $\delta^{\prime}(x) y=$ $y(0) \delta^{\prime}(x)-y^{\prime}(0) \delta(x)$ for continuously differentiable functions $y$ at the origin. Thus we may formally regard the $\delta^{\prime}$ potential as rank-two perturbation $\delta^{\prime}(x) y=\langle\delta(x), y\rangle \delta^{\prime}(x)+\left\langle\delta^{\prime}(x), y\right\rangle \delta(x)$. However, both the heuristic operators have generally no mathematical meaning. So it is not surprising that different regularizations of the distributions in (1.2) lead to different self-adjoint operators in the limit. Therefore, the pseudoHamiltonians (1.2) can be regarded as a symbolic notation only for a wide variety of quantum systems with quite different properties depending on the shape of the short-range potentials.

Recently a class of the Schrödinger operators with piecewise constant $\delta^{\prime}$-potentials were studied by Zolotaryuk [5-8]; the resonances in the transmission probability for the scattering problem were established. As was shown in Golovaty et al. [9], Golovaty and Hryniv [10, 11], and Man'ko [12] these resonances deal with the existence of zero-energy resonances and the half-bound states for singular localized potentials. The zero-energy resonances have a profound effect on the limiting behavior of the Schrödinger operators with $\delta^{\prime}$-potentials. Such operators also arose in connection with the approximation of smooth planar quantum waveguides by quantum graph [13-15]; a similar resonance phenomenon was obtained. The reader also interested in the literature on other aspects of $\delta^{\prime}$-potentials and $\delta^{\prime}$-interactions as well as approximations of point interactions by local and non-local perturbations is referred to Albeverio and Nizhnik [16], Albeverio et al. [17, 18], Exner and Manko [19], Gadella et al. [20-22].

It is known that one dimensional Schrödinger operators

$$
H(b)=\left(i \frac{d}{d x}+b(x)\right)^{2}+V(x)
$$

with continuous magnetic potentials are not especially interesting, because any continuous field $b$ is equivalent under a smooth gauge transformation to 0 . This means that the operator $H(b)$ with a continuous gauge field is unitarily equivalent to the Schrödinger operator $H(0)=-\frac{d^{2}}{d x^{2}}+V(x)$ without a magnetic field. The authors of Coutinho et al. [23] have even asserted that the phase parameter $\varphi$ in conditions (1.1) is redundant and it produces no interesting effect. They have stated that if the time-reversal invariance is imposed, the number of the parameters that specify the interactions (1.1) can be reduced to three.

For the case of singular magnetic potentials, however, there are certain nontrivial examples [24], pointing out that this case is more subtle. Albeverio et al. [24] have shown that the phase parameter is not redundant if non-stationary problems are concerned. The phase parameter can be interpreted as the amplitude of a singular gauge field. As stated in Kurasov [25] a nonzero phase $\varphi$ in the coupling conditions (1.1) may appear if and only if the singular gauge field is present. However, it is noteworthy that the factor $e^{i \varphi}$ also appeared in the solvable model for the Schrödinger operators without a magnetic field that is perturbed by a rank-two operator [26]. We also want to note that Theorem 2 in the present paper gives an example of an exactly solvable model in which the magnetic field has an effect on all coefficients $c_{k l}$ in (1.1), not only on factor $e^{i \varphi}$.

Another reason to study the 1-D Schrödinger operators with magnetic fields comes from the quantum graph theory which is a useful tool in modeling numerous physical phenomena. One of the fundamental questions of this theory consists of justifying the possibility of approximating dynamics of a quantum particle confined to real-world mesoscopic waveguides of small width $d$ by its dynamics on the graph obtained in the limit as $d$ vanishes. In Exner et al. [27], the authors demonstrated that any selfadjoint coupling in a quantum graph vertex can be approximated by a family of magnetic Schrödinger operators on a tubular network built over the graph.

The magnetic Schrödinger operators and the Dirac Hamiltonians with Aharonov-Bohm fields have been discussed from various aspects by many authors. We confine ourself to a brief overview of the most relevant papers. For the mathematical foundation of the magnetic Schrödinger operators we refer the reader to the paper of Avron et al. [28]. In two dimension, the norm resolvent convergence of the Schrödinger operators

$$
\mathcal{H}_{\varepsilon}=\left(i \nabla+\varepsilon^{-1} A(x / \varepsilon)\right)^{2}+\varepsilon^{-2} V(x / \varepsilon)
$$

with singularly scaled magnetic and electric potentials was studied by Tamura [29]. The magnetic potential had the $\delta$-like field $\varepsilon^{-2} b(x / \varepsilon)=\varepsilon^{-1} \nabla \times A(x / \varepsilon)$, and $b$ and $V$ were smooth vector functions in $\mathbb{R}^{2}$ of compact support. The limit operator strongly depends on the total flux of magnetic field and on the resonance space at zero energy. The scattering by a magnetic field with small support and the convergence to the scattering amplitude by $\delta$ magnetic field were studied in Tamura [30]. In Tamura [31], the case of relativistic particles moving in the Aharonov-Bohm magnetic field with a $\delta$-like singularity was considered. The author approximated the point-like field by smooth ones and found the limit self-adjoint operators uniquely specified by physically and mathematically reasonable boundary conditions at the origin.

The present paper can be viewed as a natural continuation of our previous works [26, 32-34], in which the case without of a magnetic field was treated.

\section{STATEMENT OF PROBLEM AND MAIN RESULTS}

Let us consider the Schrödinger operator

$$
H_{0}=-\frac{d^{2}}{d x^{2}}+V_{0}
$$


in $L_{2}(\mathbb{R})$, where potential $V_{0}$ is real-valued, measurable and locally bounded. We also assume that $V_{0}$ is bounded from below in $\mathbb{R}$. We turn now to our primary task of studying the limit behavior of two families of operators in $L_{2}(\mathbb{R})$, which can be treated as perturbations of $H_{0}$.

\subsection{Hamiltonians With Localized Potentials}

First we consider the self-adjoint operators

$$
\mathcal{H}_{\varepsilon v}=\left(i \frac{d}{d x}+\frac{1}{\varepsilon} A\left(\frac{x}{\varepsilon}\right)\right)^{2}+V_{0}(x)+\frac{\alpha}{\varepsilon^{2}} V\left(\frac{x}{\varepsilon}\right)+\frac{1}{v} U\left(\frac{x}{v}\right),
$$

where $\varepsilon$ and $v$ are small positive parameters, and $\alpha$ is a real coupling constant. Let $A, V$, and $U$ be real-valued, measurable, and bounded functions of compact support. Suppose furthermore that $A \in \mathcal{A C}(\mathbb{R})$. The domain of $\mathcal{H}_{\varepsilon v}$ coincides with dom $H_{0}$, because the perturbation has a compact support. Note that we consciously equipped potential $V$ only with a coupling constant. As we will see later, the limit behavior of $\mathcal{H}_{\varepsilon v}$ crucially depends on $\alpha$.

The potentials $\alpha \varepsilon^{-2} V\left(\varepsilon^{-1} x\right)+v^{-1} U\left(v^{-1} x\right)$ converge, as $\varepsilon$ and $v$ go to zero, to a distribution having the form $b_{1} \delta^{\prime}(x)+$ $b_{0} \delta(x)$, if $V$ has a zero-mean value, and they diverge otherwise. Hence parameter $\varepsilon$ describes the rate of shrinking for the $\delta^{\prime}$-like potential (as well as the magnetic potential), while $v$ is the rate of shrinking for the $\delta$-like potential. The sequence $\varepsilon^{-1} A\left(\varepsilon^{-1} x\right)$ converges to $\mu \delta(x)$ as $\varepsilon \rightarrow 0$ in the sense of distributions, where

$$
\mu=\int_{\mathbb{R}} A(x) d x
$$

In the partial cases, operators $\mathcal{H}_{\varepsilon v}$ can be regarded as a regularization of the first pseudo-Hamiltonian in (1.2).

Let us introduce some characteristics of the potentials $V$ and $U$.

Definition 1. We say that the Schrödinger operator $-\frac{d^{2}}{d x^{2}}+\alpha V$ in $L_{2}(\mathbb{R})$ possesses a zero-energy resonance if there exists a non trivial solution $v_{\alpha}: \mathbb{R} \rightarrow \mathbb{R}$ of the equation $-v^{\prime \prime}+\alpha V v=0$ that is bounded on the whole line. We call $v_{\alpha}$ the half-bound state of $\alpha V$.

We will simply say that the potential $\alpha V$ is resonant and it possesses a half-bound state $v_{\alpha}$. Let us denote by $\mathcal{R}(V)$ the set of all coupling constants $\alpha$ for which the potential $\alpha V$ is resonant, and introduce the mapping $\theta: \mathcal{R}(V) \rightarrow \mathbb{R}$ defined by

$$
\theta(\alpha)=\frac{v_{\alpha}^{+}}{v_{\alpha}^{-}}
$$

where $v_{\alpha}^{-}=\lim _{x \rightarrow-\infty} v_{\alpha}(x)$ and $v_{\alpha}^{+}=\lim _{x \rightarrow+\infty} v_{\alpha}(x)$. Let $\Lambda=$ $[0,+\infty]$ be the set containing the point $+\infty$.

We also define the mapping $\gamma: \mathcal{R}(V) \times \Lambda \rightarrow \mathbb{R}$ as follows:

$$
\begin{aligned}
& \gamma(\alpha, 0)=\frac{v_{\alpha}^{2}(0)}{v_{\alpha}^{-} v_{\alpha}^{+}} \int_{\mathbb{R}} U d t, \\
& \gamma(\alpha, \lambda)=\frac{1}{v_{\alpha}^{-} v_{\alpha}^{+}} \int_{\mathbb{R}} U(t) v_{\alpha}^{2}(\lambda t) d t \quad \text { for } \lambda \in(0,+\infty), \\
& \gamma(\alpha,+\infty)=\theta(\alpha) \int_{\mathbb{R}_{+}} U d t+\theta(\alpha)^{-1} \int_{\mathbb{R}_{-}} U d t
\end{aligned}
$$

We follow the notation used in Golovaty [33]. This mapping describes different kinds of the resonance interactions between the potentials $\alpha V$ and $U$ in the limit. Both the mappings $\theta$ and $\gamma$ are well defined as we will show below in Lemma 1 .

Let us introduce the subspace $\mathcal{V}$ in $L_{2}(\mathbb{R})$ as follows. We say that $h$ belongs to $\mathcal{V}$ if there exist two functions $h_{-}$and $h_{+}$ belonging to dom $H_{0}$ such that $h(x)=h_{-}(x)$ for $x<0$ and $h(x)=h_{+}(x)$ for $x>0$.

Theorem 1. Suppose that a sequence $\left\{v_{\varepsilon}\right\}_{\varepsilon>0}$ of positive numbers is such that $v_{\varepsilon} \rightarrow 0$ and ratio $v_{\varepsilon} / \varepsilon$ tends to $\lambda \in \Lambda$ as $\varepsilon \rightarrow 0$, i.e., this ratio has a finite or infinite limit. If $\alpha \in \mathcal{R}(V)$, then family of operators $\mathcal{H}_{\varepsilon v_{\varepsilon}}$ converges in the strong resolvent sense as $\varepsilon \rightarrow 0$ to the operator $\mathcal{H}=\mathcal{H}(\alpha, \lambda)$ defined by $\mathcal{H} \phi=-\phi^{\prime \prime}+V_{0} \phi$ on functions $\phi$ in $\mathcal{V}$ subject to the conditions

$$
\left(\begin{array}{c}
\phi(+0) \\
\phi^{\prime}(+0)
\end{array}\right)=e^{i \mu}\left(\begin{array}{cc}
\theta(\alpha) & 0 \\
\gamma(\alpha, \lambda) & \theta(\alpha)^{-1}
\end{array}\right)\left(\begin{array}{c}
\phi(-0) \\
\phi^{\prime}(-0)
\end{array}\right) .
$$

By analogy with the results in Golovaty [33], if potential $\alpha V$ is not resonant, the limit operator is the direct sum of two Dirichlet operators acting in $L_{2}(-\infty, 0)$ and $L_{2}(0,+\infty)$; that is, coupling conditions (2.7) must be substituted by the Dirichlet condition $\phi(0)=0$.

It is worth noting that explicit relations (2.3)-(2.6) between the matrix entries $\theta(\alpha), \gamma(\alpha, \lambda)$ and potentials $V$ and $U$ make it possible to carry out a quantitative analysis of this quantum system, e.g., to compute approximate values of the scattering data.

\subsection{Hamiltonians With Localized Rank-Two Perturbations}

We now turn our attention to another family of operators

$$
\mathcal{T}_{\varepsilon}=\left(i \frac{d}{d x}+\frac{1}{\varepsilon} A\left(\frac{x}{\varepsilon}\right)\right)^{2}+V_{0}(x)+\frac{1}{\varepsilon^{3}} F_{\varepsilon}+\frac{1}{\varepsilon} U\left(\frac{x}{\varepsilon}\right),
$$

where $F_{\varepsilon}=F_{\varepsilon}\left(f_{1}, f_{2}\right)$ are rank-two operators having the form

$$
\begin{aligned}
\left(F_{\varepsilon} \phi\right)(x) & =\bar{\beta}\left\langle f_{2}\left(\varepsilon^{-1} \cdot\right), \phi\right\rangle f_{1}\left(\frac{x}{\varepsilon}\right)+\beta\left\langle f_{1}\left(\varepsilon^{-1} \cdot\right), \phi\right\rangle f_{2}\left(\frac{x}{\varepsilon}\right) \\
& =\int_{\mathbb{R}}\left(\bar{\beta} f_{1}\left(\frac{x}{\varepsilon}\right) \bar{f}_{2}\left(\frac{s}{\varepsilon}\right)+\beta \bar{f}_{1}\left(\frac{s}{\varepsilon}\right) f_{2}\left(\frac{x}{\varepsilon}\right)\right) \phi(s) d s .
\end{aligned}
$$

Here $\langle\cdot, \cdot\rangle$ is the inner scalar product $L_{2}(\mathbb{R})$. From now on, the norm in $L_{2}(\mathbb{R})$ will be denoted by $\|\cdot\|$. Operators $\mathcal{T}_{\varepsilon}$ can be viewed as a regularization of the second pseudo-Hamiltonian in (1.2). Assume that $f_{1}, f_{2}$ and $q$ are measurable and bounded functions of compact support and $\beta$ is a complex coupling constant. The potential $q$ is real-valued.

Let us also consider rank-two perturbation of the free the Schrödinger operator

$$
B=-\frac{d^{2}}{d x^{2}}+\bar{\beta}\left\langle h_{2}, \cdot\right\rangle h_{1}+\beta\left\langle h_{1}, \cdot\right\rangle h_{2}, \quad \operatorname{dom} B=W_{2}^{2}(\mathbb{R}),
$$

where $h_{1}$ and $h_{2}$ are functions of compact support. 
Definition 2. We say that operator $B$ possesses a zeroenergy resonance provided there exists a nontrivial solution of the equation

$$
-v^{\prime \prime}+\bar{\beta}\left\langle h_{2}, v\right\rangle h_{1}+\beta\left\langle h_{1}, v\right\rangle h_{2}=0
$$

that is bounded on the whole line. This solution is called a halfbound state of $B$. We also say that $B$ admits a double zeroenergy resonance, if there exist two linearly independent halfbound states.

We will denote by $\mathcal{R}\left(h_{1}, h_{2}\right)$ the set of all coupling constants $\beta$, for which operator $B$ admits a double zero-energy resonance.

Let $h^{(-1)}$ and $h^{(-2)}$ be the first and second antiderivatives

$$
h^{(-1)}(x)=\int_{-\infty}^{x} h(s) d s, \quad h^{(-2)}(x)=\int_{-\infty}^{x}(x-s) h(s) d s
$$

for functions of compact support. Note if $h$ has zero mean, then $h^{(-1)}$ is also a function of compact support. Also, we set

$$
a(x)=\int_{-\infty}^{x} A(t) d t .
$$

Let us introduce notation

$$
g_{k}=e^{-i a} f_{k}, \quad n_{k}=\left\|g_{k}^{(-1)}\right\|, \quad p=\left\langle g_{1}^{(-1)}, g_{2}^{(-1)}\right\rangle
$$

provided $g_{1}$ and $g_{2}$ are functions of zero mean values. Therefore $n_{k}$ and $p$ are well defined, since $g_{k}^{(-1)}$ are functions of compact support. Let

$$
\omega_{\beta}=e^{i \arg \left(\beta^{-1}+p\right)} n_{2} g_{1}^{(-2)}-n_{1} g_{2}^{(-2)} .
$$

Function $\omega_{\beta}$ is constant outside some compact set containing the supports of $f_{k}$. Of course $\omega_{\beta}(x)=0$ for negative $x$ with the large absolute value. Write

$$
\varkappa=\lim _{x \rightarrow+\infty} \omega_{\beta}(x)
$$

In the case of the double zero-energy resonance function $\omega_{\beta}$ is a half-bound state of $B$ with $h_{k}=g_{k}$ (see Lemma 2 below). We also set

$$
a_{0}=\int_{\mathbb{R}} U d x, \quad a_{1}=\int_{\mathbb{R}} U \omega_{\beta} d x, \quad a_{2}=\int_{\mathbb{R}} U\left|\omega_{\beta}\right|^{2} d x .
$$

Theorem 2. Assume that $f_{1}$ and $f_{2}$ are linearly independent, $e^{-i a} f_{1}$ and $e^{-i a} f_{2}$ have zero means, and $\beta \in \mathcal{R}\left(e^{-i a} f_{1}, e^{-i a} f_{2}\right)$. Suppose also that $a_{2} \neq \bar{x} a_{1}$. Then operator family $\mathcal{T}_{\varepsilon}$ converges as $\varepsilon \rightarrow 0$ in the strong resolvent sense to operator $\mathcal{T}$ defined by $\mathcal{T} \phi=-\phi^{\prime \prime}+V_{0} \phi$ on functions $\phi$ in $\mathcal{V}$ subject to the conditions

$$
\begin{aligned}
& \left(\begin{array}{c}
\phi(+0) \\
\phi^{\prime}(+0)
\end{array}\right)=e^{i\left(\mu-\arg \left(a_{2}-\bar{\varkappa} a_{1}\right)\right)} \\
& \left(\begin{array}{cc}
\frac{a_{0}|\varkappa|^{2}-2 \operatorname{Re}\left(\bar{\varkappa} a_{1}\right)+a_{2}}{\left|a_{2}-\bar{\varkappa} a_{1}\right|} & \frac{|\varkappa|^{2}}{\left|a_{2}-\bar{\varkappa} a_{1}\right|} \\
\frac{a_{0} a_{2}-\left|a_{1}\right|^{2}}{\left|a_{2}-\bar{\varkappa} a_{1}\right|} & \frac{a_{2}}{\left|a_{2}-\bar{\varkappa} a_{1}\right|}
\end{array}\right)\left(\begin{array}{c}
\phi(-0) \\
\phi^{\prime}(-0)
\end{array}\right) .
\end{aligned}
$$

Note in these conditions that parameters $a_{1}, a_{2}$ and $\varkappa$ depend nonlinearly on coupling constant $\beta$ as well as functions $f_{1}, f_{2}, a$ via $\omega_{\beta}$; all elements of the matrix are real, since $a_{0}$ and $a_{2}$ are real number. The limit operator $\mathcal{T}$ is self-adjoint, because the determinant of matrix in (2.13) is equal to 1 [cf. (1.1)]. In fact,

$$
\begin{gathered}
\left|a_{2}-\bar{\varkappa} a_{1}\right|^{-2} \operatorname{det}\left(\begin{array}{cc}
a_{0}|\varkappa|^{2}-2 \operatorname{Re}\left(\bar{\varkappa} a_{1}\right)+a_{2} & |\varkappa|^{2} \\
a_{0} a_{2}-\left|a_{1}\right|^{2} & a_{2}
\end{array}\right) \\
=\left|a_{2}-\bar{\varkappa} a_{1}\right|^{-2}\left(a_{0} a_{2}|\varkappa|^{2}-2 a_{2} \operatorname{Re}\left(\bar{\varkappa} a_{1}\right)+a_{2}^{2}-a_{0} a_{2}|\varkappa|^{2}+|\varkappa|^{2}\left|a_{1}\right|^{2}\right) \\
=\left|a_{2}-\bar{\varkappa} a_{1}\right|^{-2}\left(a_{2}^{2}-2 a_{2} \operatorname{Re}\left(\bar{\varkappa} a_{1}\right)+|\varkappa|^{2}\left|a_{1}\right|^{2}\right) \\
=\left|a_{2}-\bar{\varkappa} a_{1}\right|^{-2}\left|a_{2}-\bar{\varkappa} a_{1}\right|^{2}=1 .
\end{gathered}
$$

Though conditions (2.13) contain the full matrix, we can not assert that it is possible to approximate any point interaction (1.1) by operators $\mathcal{T}_{\varepsilon}$. For instance, such approximation does not exist for the point interactions (1.1) with matrices

$$
\left(\begin{array}{cc}
c_{11} & 0 \\
c_{21} & c_{11}^{-1}
\end{array}\right),
$$

where $c_{11}$ is different from 1 ; if $\varkappa=0$, then the matrix in (2.13) has the unit diagonal. Therefore, Theorems 1 and 2 are in some sense mutually complementary.

Remark also that for any pair of linearly independent functions $f_{1}, f_{2}$ satisfying the assumptions of the theorem there exists a wide class of potentials $U$ for which condition $a_{2} \neq$ $\bar{x} a_{1}$ holds.

In view of Theorems 1 and 2 in Golovaty [26] we can expect that there exist at least six essentially different cases of the limiting behavior for $\mathcal{T}_{\varepsilon}$ as $\varepsilon \rightarrow 0$. However, in this paper we restrict ourselves to analyzing only the case that is described in Theorem 2. Just this case covers the widest class of point interactions in the limit.

\section{ZERO-ENERGY RESONANCES AND HALF-BOUND STATES}

We show first that the set $\mathcal{R}(V)$ of all resonance coupling constants for operator $-\frac{d^{2}}{d x^{2}}+\alpha V$ is not empty and furthermore it is rich enough for any function $V$ of compact support.

Lemma 1. (i) For each measurable function $V$ of compact support, the resonant set $\mathcal{R}(V)$ is a countable subset of the real line with one or two accumulation points at infinity.

(ii) For each $\alpha \in \mathcal{R}(V)$, the corresponding half-bound state $v_{\alpha}$ is unique up to a scalar factor. Moreover, both the limits

$$
v_{\alpha}^{-}=\lim _{x \rightarrow-\infty} v_{\alpha}(x), \quad v_{\alpha}^{+}=\lim _{x \rightarrow+\infty} v_{\alpha}(x)
$$

exist and are different from zero.

Proof: Without loss of generality we assume that supp $V \subset \mathcal{I}$, where $\mathcal{I}=(-1,1)$. Then operator $-\frac{d^{2}}{d x^{2}}+\alpha V$ possesses a half-bound state if and only if the problem

$$
-v^{\prime \prime}+\alpha V v=0, \quad x \in \mathcal{I}, \quad v^{\prime}(-1)=0, \quad v^{\prime}(1)=0
$$


has a non-trivial solution. In fact, a half-bound state $v_{\alpha}$ is constant outside $\mathcal{I}$ as a bounded solution of equation $v^{\prime \prime}=0$ and hence $v_{\alpha}^{\prime}(-1)=v_{\alpha}^{\prime}(1)=0$. From this we also deduce that there exist the limits (3.1). Obviously we have $v_{\alpha}^{-}=v_{\alpha}(-1)$ and $v_{\alpha}^{+}=v_{\alpha}(1)$. In addition, both the values $v_{\alpha}(-1)$ and $v_{\alpha}(1)$ are different from zero in view of uniqueness for the Cauchy problem, because $v_{\alpha}$ is a non-trivial solution.

Problem (3.2) can be regarded as a spectral problem with spectral parameter $\alpha$. If $V$ is a function of fixed sign, then (3.2) is a standard Sturm-Liouville problem and $\mathcal{R}(V)$ coincides with the spectrum of a self-adjoint operator in weighted Lebesgue spaces $L_{2}(V, \mathcal{I})$. Otherwise, we can interpret (3.2) as the eigenvalue problem with indefinite weight function $V$; the problem can be associated with a self-adjoint non-negative operator $K$ in a Krein space $[9,32]$. In both the cases the spectra of such operators are real and discrete with accumulation points at $-\infty$ or $+\infty$ only. Moreover all nonzero eigenvalues are simple; for the case of the Krein space, $\alpha=0$ is generally semi-simple. The reader can also refers to Iohvidov et al. [35] for the details of the theory of self-adjoint operators in Krein spaces. It follows from the simplicity of spectra that half-bound state $v_{\alpha}$ is unique up to a scalar factor.

The set $\mathcal{R}\left(h_{1}, h_{2}\right)$ of coupling constants, for which the operator $B$ possesses the double zero-range resonance, is also rich for any pair of $h_{1}$ and $h_{2}$. We set $m_{k}=\left\|h_{k}^{(-1)}\right\|$ and $\tau=$ $\left\langle h_{1}^{(-1)}, h_{2}^{(-1)}\right\rangle$.

Lemma 2. Assume that $h_{1}, h_{2}$ are linearly independent functions of zero mean. Then set $\mathcal{R}\left(h_{1}, h_{2}\right)$ of double zero-range resonance for operator $B$ is the circle

$$
\mathcal{R}\left(h_{1}, h_{2}\right)=\left\{\beta \in \mathbb{C}:\left|\beta-\beta_{0}\right|=\rho\right\}
$$

in the complex plane, where

$$
\beta_{0}=\frac{\bar{\tau}}{m_{1}^{2} m_{2}^{2}-|\tau|^{2}}, \quad \rho=\frac{m_{1} m_{2}}{m_{1}^{2} m_{2}^{2}-|\tau|^{2}} .
$$

In addition, if $\beta \in \mathcal{R}\left(h_{1}, h_{2}\right)$, then the constant function and function

$$
\omega_{\beta}=e^{i \arg \left(\beta^{-1}+\tau\right)} m_{2} h_{1}^{(-2)}-m_{1} h_{2}^{(-2)}
$$

are two linearly independent half-bound states of $B$.

Note that circle $\mathcal{R}\left(h_{1}, h_{2}\right)$ is well defined for linearly independent $h_{1}$ and $h_{2}$, because then the first antiderivatives $h_{1}^{(-1)}$ and $h_{2}^{(-1)}$ are also linearly independent, and $|\tau|<m_{1} m_{2}$ in view of the Cauchy-Schwartz inequality. For instance, if functions $h_{1}^{(-1)}$ and $h_{2}^{(-1)}$ are orthonormal, then $\mathcal{R}\left(h_{1}, h_{2}\right)$ is a unit circle centered at the origin, since $m_{1}=m_{2}=1$ and $\tau=0$. If $h_{1}^{(-1)}$ and $h_{2}^{(-1)}$ are simply orthogonal, then $\mathcal{R}\left(h_{1}, h_{2}\right)=\{\beta \in$ $\left.\mathbb{C}:|\beta|=m_{1}^{-1} m_{2}^{-1}\right\}$. In the case when $h_{2}=h_{1}+\varepsilon g$ and $\varepsilon$ is small, that is to say, the angle between $h_{1}$ and $h_{2}$ is small, the center $\beta_{0}$ is far from the origin and the radius $\rho$ is large, because then the difference $m_{1} m_{2}-|\tau|$ is of order $\varepsilon$.
Proof: We start with the observation that $v=1$ is obviously a solution of equation

$$
-v^{\prime \prime}+\bar{\beta}\left\langle h_{2}, v\right\rangle h_{1}+\beta\left\langle h_{1}, v\right\rangle h_{2}=0,
$$

since $h_{k}$ are functions with zero-mean values. For the same reason, the second anti-derivatives $h_{k}^{(-2)}$ are bounded on the whole line. Then regarding this equation as the "nonhomogeneous" one

$$
v^{\prime \prime}=\bar{\beta}\left\langle h_{2}, v\right\rangle h_{1}+\beta\left\langle h_{1}, v\right\rangle h_{2},
$$

we can look for another half-bound state in the form $\omega=c_{1} h_{1}^{(-2)}+c_{2} h_{2}^{(-2)}$. We do not take into account solution $x$ of the homogeneous equation, because it is unbounded as $|x| \rightarrow \infty$.

Since $h_{1}$ and $h_{2}$ are linearly independent, substituting $\omega$ into (3.3) yields

$$
\left\{\begin{array}{l}
\beta\left\langle h_{1}, h_{1}^{(-2)}\right\rangle c_{1}+\left(\beta\left\langle h_{1}, h_{2}^{(-2)}\right\rangle-1\right) c_{2}=0, \\
\left(\bar{\beta}\left\langle h_{2}, h_{1}^{(-2)}\right\rangle-1\right) c_{1}+\bar{\beta}\left\langle h_{2}, h_{2}^{(-2)}\right\rangle c_{2}=0 .
\end{array}\right.
$$

Because $h_{j}$ has compact support, the scalar product $\left\langle h_{j}, h_{k}^{(-2)}\right\rangle$ is finite, even though antiderivative $h_{k}^{(-2)}$ does not belong to $L_{2}(\mathbb{R})$. In addition, the integrating by parts shows $\left\langle h_{j}, h_{k}^{(-2)}\right\rangle=$ $-\left\langle h_{j}^{(-1)}, h_{k}^{(-1)}\right\rangle$. Then (3.4) becomes

$$
\left\{\begin{aligned}
\beta m_{1}^{2} c_{1}+(\beta \tau+1) c_{2} & =0 \\
(\overline{\beta \tau}+1) c_{1}+\quad \bar{\beta} m_{2}^{2} c_{2} & =0
\end{aligned}\right.
$$

This system has a non-trivial solution $\left(c_{1}, c_{2}\right)$ if and only if $|\beta| m_{1} m_{2}=|\beta \tau+1|$. The condition can be written as $\left|\beta^{-1}+\tau\right|=m_{1} m_{2}$.

Given $a \in \mathbb{C}$ and $r \in \mathbb{R}$, we consider the circle $\{z \in \mathbb{C}: \mid z-$ $a \mid=r$. Suppose that $|a|<r$. The mapping $z \mapsto z^{-1}$ is a bijection from this circle onto another one

$$
\left\{z \in \mathbb{C}:\left|z+\frac{\bar{a}}{r^{2}-|a|^{2}}\right|=\frac{r}{r^{2}-|a|^{2}}\right\},
$$

as is easy to check. Therefore, the resonance region $\mathcal{R}\left(h_{1}, h_{2}\right)$ arises as the image of the circle $\left\{z \in \mathbb{C}:|z+\tau|=m_{1} m_{2}\right\}$ under the transformation $z \mapsto z^{-1}$. Note that $|\tau|<m_{1} m_{2}$ by the Cauchy-Schwartz inequality.

If $\beta \in \mathcal{R}\left(h_{1}, h_{2}\right)$, then (3.5) admits a nontrivial solution having the form

$$
c_{1}=e^{i \arg \left(\beta^{-1}+\tau\right)} m_{2}, \quad c_{2}=-m_{1} .
$$

In fact, substituting this solution into the first equation yields

$$
\begin{aligned}
\beta m_{1}^{2} c_{1}+(\beta \tau+1) c_{2} & =\beta m_{1}^{2} m_{2} e^{i \arg \left(\beta^{-1}+\tau\right)}-m_{1}(\beta \tau+1) \\
=\beta m_{1}\left|\beta^{-1}+\tau\right| e^{i \arg \left(\beta^{-1}+\tau\right)}-m_{1}(\beta \tau+1) & =\beta m_{1}\left(\beta^{-1}+\tau\right)-m_{1}(\beta \tau+1)=0
\end{aligned}
$$

since $m_{1} m_{2}=\left|\beta^{-1}+\tau\right|$. Hence, $\omega_{\beta}=e^{i \arg \left(\beta^{-1}+\tau\right)} m_{2} h_{1}^{(-2)}-$ $m_{1} h_{2}^{(-2)}$ is a half-bound state of $B$, which is different from the constant one. 


\section{PROOF OF MAIN RESULTS}

We start with some assertions, which will be used below.

Lemma 3. Let $\left\{S_{\varepsilon}\right\}_{\varepsilon>0}$ be a family of self-adjoint operators in a Hilbert space $\mathcal{L}$ and $\left\{W_{\varepsilon}\right\}_{\varepsilon>0}$ be a family of unitary operators in $\mathcal{L}$. Assume that $S_{\varepsilon} \rightarrow S$ as $\varepsilon \rightarrow 0$ in the norm resolvent sense, $W_{\varepsilon} \rightarrow W$ in the strong operator topology as $\varepsilon \rightarrow 0$ and $W$ is a unitary operator in $\mathcal{L}$. Then the family of operators $Q_{\varepsilon}=$ $W_{\varepsilon} S_{\varepsilon} W_{\varepsilon}^{-1}$ converges in the strong resolvent sense to the operator $Q=W S W^{-1}$ with the domain $\left\{\phi \in \mathcal{L}: W^{-1} \phi \in \operatorname{dom} S\right\}$.

Proof: We first note that

$$
\begin{aligned}
& \left(Q_{\varepsilon}-\zeta\right)^{-1}-(Q-\zeta)^{-1}=W_{\varepsilon}\left(\left(S_{\varepsilon}-\zeta\right)^{-1}-(S-\zeta)^{-1}\right) W_{\varepsilon}^{-1} \\
& +W_{\varepsilon}(S-\zeta)^{-1}\left(W_{\varepsilon}^{-1}-W^{-1}\right)+\left(W_{\varepsilon}-W\right)(S-\zeta)^{-1} W^{-1}
\end{aligned}
$$

provided $\zeta \in \mathbb{C} \backslash \mathbb{R}$. The operator $S$ is self-adjoint as a limit of self-adjoint operators $S_{\varepsilon}$ in the norm resolvent topology. From the last relation and the self-adjointness of $S$ we have

$$
\begin{gathered}
\left\|\left(Q_{\varepsilon}-\zeta\right)^{-1} f-(Q-\zeta)^{-1} f\right\| \leq\left\|\left(S_{\varepsilon}-\zeta\right)^{-1}-(S-\zeta)^{-1}\right\|\|f\| \\
+|\operatorname{Im} \zeta|^{-1}\left\|\left(W_{\varepsilon}^{-1}-W^{-1}\right) f\right\|+\left\|\left(W_{\varepsilon}-W\right)(S-\zeta)^{-1} W^{-1} f\right\|
\end{gathered}
$$

for all $f \in \mathcal{L}$. The first term in the right-hand side tends to zero as $\varepsilon \rightarrow 0$, since operators $S_{\varepsilon}$ converge to $S$ in the norm resolvent sense. The last two terms are infinitely small as $\varepsilon \rightarrow 0$, in view of the strong convergence of $W_{\varepsilon}$.

We introduce two unitary operators

$$
\left(W_{\varepsilon} f\right)(x)=e^{i a\left(\frac{x}{\varepsilon}\right)} f(x), \quad(W f)(x)=e^{i \mu H(x)} f(x),
$$

in $L_{2}(\mathbb{R})$, where $a$ and $\mu$ given by (2.11) and (2.2), respectively, and $H$ is the Heaviside step function

$$
H(x)= \begin{cases}0, & \text { for } x<0 \\ 1, & \text { for } x>0\end{cases}
$$

Lemma 4. Let $W_{\varepsilon}$ and $W$ be the unitary operators given by (4.2). Then $W_{\varepsilon}$ converge to $W$ as $\varepsilon \rightarrow 0$ in the strong operator topology.

Proof: Without loss of generality we can assume that the support of the magnetic potential $A$ lies in $(-1,1)$. Therefore, $a\left(\varepsilon^{-1} x\right)=0$ for $x<-\varepsilon$ and $a\left(\varepsilon^{-1} x\right)=\mu$ for $x>\varepsilon$. For each $f \in L_{2}(\mathbb{R})$ we have

$$
\begin{aligned}
& \left\|W_{\varepsilon} f-W f\right\|^{2} \leq \int_{\mathbb{R}}\left|e^{i a\left(\frac{x}{\varepsilon}\right)}-e^{i \mu H(x)}\right|^{2}|f(x)|^{2} d x \\
& =\int_{-\varepsilon}^{\varepsilon}\left|e^{i a\left(\frac{x}{\varepsilon}\right)}-e^{i \mu H(x)}\right|^{2}|f(x)|^{2} d x \leq 4 \int_{-\varepsilon}^{\varepsilon}|f(x)|^{2} d x,
\end{aligned}
$$

since $a\left(\varepsilon^{-1} x\right)=\mu H(x)$ for $|x|>\varepsilon$. The right-hand side of (4.3) tends to zero as $\varepsilon \rightarrow 0$, by absolute continuity of the Lebesgue integral.

\subsection{Proof of Theorem 1}

Let us consider the Schrödinger operators

$$
\begin{gathered}
S_{\varepsilon}=-\frac{d^{2}}{d x^{2}}+V_{0}(x)+\frac{\alpha}{\varepsilon^{2}} V\left(\frac{x}{\varepsilon}\right)+\frac{1}{v_{\varepsilon}} U\left(\frac{x}{v_{\varepsilon}}\right), \\
\operatorname{dom} S_{\varepsilon}=W_{2}^{2}(\mathbb{R}) .
\end{gathered}
$$

It is of course that $S_{\varepsilon}$ is a version of operator $\mathcal{H}_{\varepsilon v}$ given by (2.1) when the magnetic potential is disabled. We also denote by $S=$ $S(\theta, \gamma)$ the Schrödinger operator acting via $S \psi=-\psi^{\prime \prime}+V_{0} \psi$ on functions $\psi$ in $\mathcal{V}$ obeying the interface conditions

$$
\left(\begin{array}{c}
\psi(+0) \\
\psi^{\prime}(+0)
\end{array}\right)=\left(\begin{array}{cc}
\theta & 0 \\
\gamma & \theta^{-1}
\end{array}\right)\left(\begin{array}{c}
\psi(-0) \\
\psi^{\prime}(-0)
\end{array}\right)
$$

at the origin. For all real $\theta$ and $\gamma$, this operator is self-adjoint.

The proof of Theorem 1 is based on the results obtained in Golovaty [32, 33]. Let $\left\{v_{\varepsilon}\right\}_{\varepsilon>0}$ be a sequence such that $v_{\varepsilon} \rightarrow 0$ as $\varepsilon \rightarrow 0$ and the ratio $v_{\varepsilon} / \varepsilon$ tends to $\lambda \in \Lambda$. If the potential $\alpha V$ is resonant, then the operator family $S_{\varepsilon}$ converges in the norm resolvent sense as $\varepsilon \rightarrow 0$ to operator $S=S(\theta(\alpha), \gamma(\alpha, \lambda))$, where $\theta, \gamma$ are given by (2.3)-(2.6). We see at once that operator $\mathcal{H}_{\varepsilon v_{\varepsilon}}$ is unitarily equivalent to operator $S_{\varepsilon}$, i.e., $\mathcal{H}_{\varepsilon v_{\varepsilon}}=W_{\varepsilon} S_{\varepsilon} W_{\varepsilon}^{-1}$ with the unitary operator (the gauge transformation) $W_{\varepsilon}$ given by (4.2) [28]. For instance, it is easy to check that

$$
-e^{i a\left(\frac{x}{\varepsilon}\right)} \frac{d^{2}}{d x^{2}}\left(e^{-i a\left(\frac{x}{\varepsilon}\right)} \phi(x)\right)=\left(i \frac{d}{d x}+\frac{1}{\varepsilon} A\left(\frac{x}{\varepsilon}\right)\right)^{2} \phi(x),
$$

since $a^{\prime}=A$. Next, $W^{-1}(\operatorname{dom} \mathcal{H}) \subset \operatorname{dom} S$, where $W^{-1} f=$ $e^{-i \mu H} f$. In fact, given $\phi \in \operatorname{dom} \mathcal{H}$, we set $\psi=W^{-1} \phi=e^{-i \mu H} \phi$. Then we have $\psi(+0)=e^{-i \mu} \phi(+0), \psi^{\prime}(+0)=e^{-i \mu} \phi^{\prime}(+0)$, $\psi(-0)=\phi(-0)$ and $\psi^{\prime}(-0)=\phi^{\prime}(-0)$. Rewriting conditions (2.7) for $\phi$ in the form

$$
\left(\begin{array}{c}
e^{-i \mu} \phi(+0) \\
e^{-i \mu} \phi^{\prime}(+0)
\end{array}\right)=\left(\begin{array}{cc}
\theta(\alpha) & 0 \\
\gamma(\alpha, \lambda) & \theta(\alpha)^{-1}
\end{array}\right)\left(\begin{array}{c}
\phi(-0) \\
\phi^{\prime}(-0)
\end{array}\right)
$$

we ascertain that $\psi$ satisfies (4.5) and therefore $\psi \in$ $\operatorname{dom}$ S. Obviously,

$$
W^{-1}: \operatorname{dom} \mathcal{H} \rightarrow \operatorname{dom} S
$$

is a linear isomorphism. Therefore, the limit operator $\mathcal{H}$ in Theorem 1 can be written as $\mathcal{H}=W S W^{-1}$.

In view of Lemma 4 , the gauge transformations $W_{\varepsilon}$ converge to $W$ in the strong operator topology. Since the resolvents of $S_{\varepsilon}$ converge to the resolvent of $S$ uniformly, we deduce from Lemma 3 that

$$
\mathcal{H}_{\varepsilon} v_{\varepsilon}=W_{\varepsilon} S_{\varepsilon} W_{\varepsilon}^{-1} \rightarrow W S W^{-1}=\mathcal{H} \quad \text { as } \varepsilon \rightarrow 0
$$

in the strong resolvent sense. 


\subsection{Proof of Theorem 2}

We can now argue almost exactly as in the proof of Theorem 1. First of all note that operators $\mathcal{T}_{\varepsilon}$ given by (4.6) are unitarily equivalent to operators

$$
T_{\varepsilon}=-\frac{d^{2}}{d x^{2}}+V_{0}(x)+\frac{1}{\varepsilon^{3}} G_{\varepsilon}+\frac{1}{\varepsilon} U\left(\frac{x}{\varepsilon}\right),
$$

namely $\mathcal{T}_{\varepsilon}=W_{\varepsilon} T_{\varepsilon} W_{\varepsilon}^{-1}$ with the gauge transformation $W_{\varepsilon}$ given by (4.2). Operator $G_{\varepsilon}=G_{\varepsilon}\left(g_{1}, g_{2}\right)$ is a rank-two operator of the form

$\left(G_{\varepsilon} \psi\right)(x)=\bar{\beta}\left\langle g_{2}\left(\varepsilon^{-1} \cdot\right), \psi\right\rangle g_{1}\left(\varepsilon^{-1} x\right)+\beta\left\langle g_{1}\left(\varepsilon^{-1} \cdot\right), \psi\right\rangle g_{2}\left(\varepsilon^{-1} x\right)$,

where $g_{1}=e^{-i a} f_{1}$ and $g_{2}=e^{-i a} f_{2}$ are the same functions as in (2.12). In fact, a trivial verification shows that $F_{\varepsilon}=W_{\varepsilon} G_{\varepsilon} W_{\varepsilon}^{-1}$.

In Theorem 2 we assumed $g_{1}, g_{2}$ were linearly independent functions of zero mean. Moreover, $a_{2} \neq \bar{x} a_{1}$. It has recently been proved in Golovaty [26] that if additionally coupling constant $\beta$ belongs to the set $\mathcal{R}\left(g_{1}, g_{2}\right)$ of double zero-energy resonance for $B=-\frac{d^{2}}{d x^{2}}+\bar{\beta}\left\langle g_{2}, \cdot\right\rangle g_{1}+\beta\left\langle g_{1}, \cdot\right\rangle g_{2}$, then operators $T_{\varepsilon}$ converge as $\varepsilon \rightarrow 0$ in the norm resolvent sense to operator $T \psi=-\psi^{\prime \prime}+V_{0} \psi$ acting on functions $\psi \in \mathcal{V}$ obeying the interface conditions

$$
\begin{aligned}
& \left(\begin{array}{c}
\psi(+0) \\
\psi^{\prime}(+0)
\end{array}\right)=e^{i \arg \left(a_{2}-\varkappa \bar{a}_{1}\right)} \\
& \left(\begin{array}{cc}
\frac{|\varkappa|^{2} a_{0}-2 \operatorname{Re}\left(\bar{\varkappa} a_{1}\right)+a_{2}}{\left|a_{2}-\bar{\varkappa} a_{1}\right|} & \frac{|\varkappa|^{2}}{\left|a_{2}-\bar{\varkappa} a_{1}\right|} \\
\frac{a_{0} a_{2}-\left|a_{1}\right|^{2}}{\left|a_{2}-\bar{\varkappa} a_{1}\right|} & \frac{a_{2}}{\left|a_{2}-\bar{\varkappa} a_{1}\right|}
\end{array}\right)\left(\begin{array}{c}
\psi(-0) \\
\psi^{\prime}(-0)
\end{array}\right)
\end{aligned}
$$

at the origin. Therefore, $\mathcal{T}=W T W^{-1}$, by reasoning similar to that in the proof of Theorem 1 . We can now repeatedly apply

\section{REFERENCES}

1. Albeverio S, Gesztesy F, Høegh-Krohn R, Holden H. Solvable Models In Quantum Mechanics. Providence, RI: AMS Chelsea Publishing (2005).

2. Albeverio S, Kurasov P. Singular Perturbations of Differential Operators and Solvable Schrödinger Type Operators. Cambridge: Cambridge University Press (2000).

3. Albeverio S, Dąbrowski L, Kurasov P. Symmetries of schrödinger operators with point interactions. Lett Math Phys. (1998) 45:33-47. doi: 10.1023/A:1007493325970

4. Chernoff P, Hughes R. A new class of point interactions in one dimension. $J$ Funct Anal. (1993) 111:97-117.

5. Zolotaryuk AV. Two-parametric resonant tunneling across the $\delta^{\prime}(x)$ potential. Adv Sci Lett. (2008) 1:187-91. doi: 10.1166/asl.2008.019

6. Zolotaryuk AV. Point interactions of the dipole type defined through a threeparametric power regularization. J Phys A Math Theor. (2010) 43:105302. doi: 10.1088/1751-8113/43/10/105302

7. Zolotaryuk AV, Zolotaryuk Y. Controlling a resonant transmission across the $\delta^{\prime}(x)$-potential: the inverse problem. J Phys A Math Theor. (2011) 44:375305. doi: $10.1088 / 1751-8113 / 44 / 37 / 375305$

8. Zolotaryuk AV. Families of one-point interactions resulting from the squeezing limit of the sum of two- and three-delta-like potentials. J Phys A Math Theor. (2017) 50:225303. doi: 10.1088/1751-8121/aa6dc2

9. Golovaty Y, Man'ko S. Solvable models for the Schrödinger operators with $\delta^{\prime}$ like potentials. Ukr Math Bull. (2009) 6:169-203. Available online at: https:// arxiv.org/abs/0909.1034
Lemma 3 for operator families $\left\{T_{\varepsilon}\right\}_{\varepsilon>0}$ and $\left\{W_{\varepsilon}\right\}_{\varepsilon>0}$ to deduce the strong resolvent convergence

$$
\mathcal{T}_{\varepsilon}=W_{\varepsilon} T_{\varepsilon} W_{\varepsilon}^{-1} \rightarrow W T W^{-1}=\mathcal{T}
$$

as $\varepsilon \rightarrow 0$.

\section{FINAL REMARKS}

In Theorem 1 we obtained in the limit the coupling conditions

$$
\left(\begin{array}{c}
\phi(+0) \\
\phi^{\prime}(+0)
\end{array}\right)=e^{i \mu(A)}\left(\begin{array}{cc}
\theta(V) & 0 \\
\gamma(V, U) & \theta^{-1}(V)
\end{array}\right)\left(\begin{array}{c}
\phi(-0) \\
\phi^{\prime}(-0)
\end{array}\right),
$$

in which the magnetic potential $A$ appeared only in the phase factor $e^{i \mu(A)}$. This situation is typical for potential perturbations of Schrödinger operators.

Unlike the previous case, in which the potential perturbation was invariant with respect to the gauge transformation $W_{\varepsilon}$, the finite-rank perturbation $F_{\varepsilon}$ is not invariant. In fact, $F_{\varepsilon}=$ $W_{\varepsilon} G_{\varepsilon} W_{\varepsilon}^{-1}$; transformation $W_{\varepsilon}$ rotates the plane $\operatorname{span}\left\{f_{1}, f_{2}\right\}$ when we change parameter $\varepsilon$. This is certainly the main reason why the magnetic field $A$ has an effect on all coefficients in the coupling conditions

$$
\left(\begin{array}{c}
\phi(+0) \\
\phi^{\prime}(+0)
\end{array}\right)=e^{i \mu(A)}\left(\begin{array}{ll}
c_{11}(A) & c_{12}(A) \\
c_{21}(A) & c_{22}(A)
\end{array}\right)\left(\begin{array}{c}
\phi(-0) \\
\phi^{\prime}(-0)
\end{array}\right)
$$

appearing as the solvable model in Theorem 2. Of course, the coefficients $c_{k j}$ depend on potentials $V, U$ and functions $f_{1}, f_{2}$ too.

\section{AUTHOR CONTRIBUTIONS}

The author confirms being the sole contributor of this work and has approved it for publication.

10. Golovaty Y, Hryniv R. On norm resolvent convergence of Schrödinger operators with $\delta^{\prime}$-like potentials. J Phys A Math Theor. (2010) 43:155204. doi: 10.1088/1751-8113/43/15/155204

11. Golovaty YD, Hryniv RO. Norm resolvent convergence of singularly scaled Schrödinger operators and $\delta^{\prime}$-potentials. Proc R Soc Sec A Math. (2013) 143:791-816. doi: 10.1017/S0308210512000194

12. Man'ko SS. On $\delta^{\prime}$-like potential scattering on star graphs. J Phys A Math Theor. (2010) 43:445304. doi: 10.1088/1751-8113/43/44/ 445304

13. Albeverio S, Cacciapuoti C, Finco D. Coupling in the singular limit of thin quantum waveguides. J Math Phys. (2007) 48:032103. doi: 10.1063/ 1.2710197

14. Cacciapuoti C, Exner P. Nontrivial edge coupling from a Dirichlet network squeezing: the case of a bent waveguide. J Phys A Math Theor. (2007) 40:F511-23. doi: 10.1088/1751-8113/40/26/F02

15. Cacciapuoti C, Finco D. Graph-like models for thin waveguides with Robin boundary conditions. Asympt Anal. (2010) 70:199-230. doi: 10.3233/ASY-2010-1014

16. Albeverio S, Nizhnik L. Schrödinger operators with nonlocal potentials. Methods Funct Anal Topol. (2013) 19:199-210. Available online at: http:// mfat.imath.kiev.ua/article/?id=698

17. Albeverio S, Fassari S, Rinaldi F. A remarkable spectral feature of the Schrödinger Hamiltonian of the harmonic oscillator perturbed by an attractive $\delta^{\prime}$-interaction centred at the origin: double degeneracy and level crossing. J Phys A Math Theor. (2013) 46:385305. doi: $10.1088 / 1751-8113 / 46 / 38 / 385305$ 
18. Albeverio S, Fassari S, Rinaldi F. The Hamiltonian of the harmonic oscillator with an attractive $\delta^{\prime}$-interaction centred at the origin as approximated by the one with a triple of attractive $\delta$-interactions. J Phys A Math Theor. (2015) 49:025302. doi: 10.1088/1751-8113/49/2/025302

19. Exner P, Manko S. Approximations of quantum-graph vertex couplings by singularly scaled rank-one operators. Lett Math Phys. (2014) 104:1079-94. doi: 10.1007/s11005-014-0706-1

20. Gadella M, Negro J, Nieto LM. Bound states and scattering coefficients of the $-a \delta(x)+b \delta^{\prime}(x)$ potential. Phys Lett A. (2009) 373:1310-3. doi: $10.1016 /$ j.physleta.2009.02.025

21. Gadella M, Glasser ML, Nieto LM. One dimensional models with a singular potential of the type $-a \delta(x)+b \delta^{\prime}(x)$. Int J Theor Phys. (2011) 50:2144-52. doi: 10.1007/s10773-010-0641-6

22. Gadella M, García-Ferrero MA, González-Martin S, Maldonado-Villamizar FH. The infinite square well with a point interaction: a discussion on the different parameterizations. Int J Theor Phys. (2014) 53:1614. doi: 10.1007/s10773-013-1959-7

23. Coutinho FAB, Nogami Y, Tomio L. Time-reversal aspect of the point interactions in one-dimensional quantum mechanics. J Phys A. (1999) 32:L133-6.

24. Albeverio S, Fei S-M, Kurasov P. Gauge fields, point interactions and few-body problems in one dimension. Rep Math Phys. (2004) 53:363-70. doi: 10.1016/S0034-4877(04)90023-7

25. Kurasov P. Distribution theory for discontinuous test functions and differential operators with generalized coefficients. J Math Anal Appl. (1996) 201:297-323.

26. Golovaty Y. Schrödinger operators with singular rank-two perturbations and point interactions. Integr Equat Oper Theory. (2018) 90:57. doi: 10.1007/s00020-018-2482-2

27. Exner P, Post O. A general approximation of quantum graph vertex couplings by scaled Schrödinger operators on thin branched manifolds. Commun Math Phys. (2013) 322:207. doi: 10.1007/s00220-013-1699-9
28. Avron J, Herbst I, Simon B. Schrödinger operators with magnetic fields. I. General interactions. Duke Math J. (1978) 45:847-83.

29. Tamura H. Norm resolvent convergence to magnetic Schrödinger operators with point interactions. Rep Math Phys. (2001) 13:465-511. doi: 10.1142/S0129055X01000697

30. Tamura H. Magnetic scattering at low energy in two dimensions. Nagoya Math J. (1999) 155:95-151.

31. Tamura H. Resolvent convergence in norm for Dirac operator with AharonovBohm field. J Math Phys. (2003) 44:2967. doi: 10.1063/1.1580200

32. Golovaty Y. Schrödinger operators with $\left(\alpha \delta^{\prime}+\beta \delta\right)$-like potentials: norm resolvent convergence and solvable models. Methods Funct Anal Topol. (2012) 18:243-55. Available online at: http://mfat.imath.kiev.ua/article/?id=633

33. Golovaty Y. 1D Schrödinger operators with short range interactions: two-scale regularization of distributional potentials. Integr Equat Oper Theory. (2013) 75:341-62. doi: 10.1007/s00020-012-2027-z

34. Golovaty Y. Two-parametric $\delta^{\prime}$-interactions: approximation by Schrödinger operators with localized rank-two perturbations. J Phys A Math Theor. (2018) 51:255202. doi: 10.1088/1751-8121/aac110

35. Iohvidov IS, Krein MG, Langer H. Introduction to the Spectral Theory of Operators in Spaces With an Indefinite Metric. Berlin: Akademie-Verlag (1982).

Conflict of Interest Statement: The author declares that the research was conducted in the absence of any commercial or financial relationships that could be construed as a potential conflict of interest.

Copyright (C) 2019 Golovaty. This is an open-access article distributed under the terms of the Creative Commons Attribution License (CC BY). The use, distribution or reproduction in other forums is permitted, provided the original author(s) and the copyright owner(s) are credited and that the original publication in this journal is cited, in accordance with accepted academic practice. No use, distribution or reproduction is permitted which does not comply with these terms. 